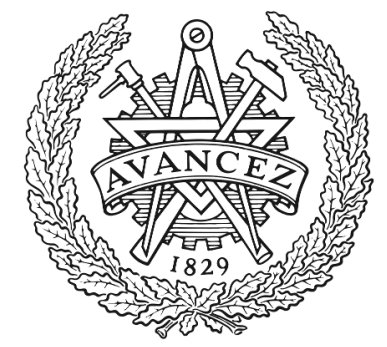

CHALMERS

UNIVERSITY OF TECHNOLOGY

\title{
Influence of front/rear drive force distribution on the lateral grip and understeer of all-wheel drive vehicles
}

Downloaded from: https://research.chalmers.se, 2023-04-26 07:10 UTC

Citation for the original published paper (version of record):

Klomp, M., Thomson, R. (2011). Influence of front/rear drive force distribution on the lateral grip and understeer of

all-wheel drive vehicles. International Journal of Vehicle Design, 56(1/2/3/4): 34-48.

http://dx.doi.org/10.1504/IJVD.2011.043272

N.B. When citing this work, cite the original published paper. 


\title{
Influence of Front/Rear Drive Force Distribution on the Lateral Grip and Understeer of All-Wheel Drive Vehicles
}

\author{
M. Klomp* \\ Saab Automobile \\ SE-461 80, Trollhättan Sweden \\ R. Thomson \\ Swedish National Road and Transport Research Institute \\ SE-402 78 Gothenburg, Sweden
}

April 19, 2010

\begin{abstract}
The increased control potential in all-wheel drive vehicles enables the vehicle to maintain understeer characteristics from the linear tire range up to the grip limit. This provides consistent feedback to the driver over a wider range of operating conditions than for two-wheel drive vehicles. In order to aid the development of current and emerging driveline systems, the authors see a need for improved theory and methods (numerical and graphical) describing the influence of drive force distribution on these said factors. In this work the methods for computing the lateral grip margin for a general drive force distribution are developed for four special cases; front-wheel drive, rear-wheel drive, rigid all-wheel drive; and finally, an optimal front/rear drive force distribution.
\end{abstract}

\section{Introduction}

Currently evolving advanced all-wheel drive (AWD) systems provide opportunities to actively control each individual wheel's drive torque [7. The increased number of control inputs, when compared to traditional driveline configurations, enables the vehicle to maintain understeer characteristics from the linear tire operating range up to the grip limit. Without a fixed drive force distribution, the lateral grip will be determined by the front or rear axle depending on the operating range. This may cause inconsistent understeer characteristics for some instances or even change to oversteer. Changes in the understeer characteristics of the vehicle can, however, also be an important feedback for the driver that can be eliminated through full AWD control.

Two important steady-state handling characteristics for road vehicles are the lateral grip limit, here defined as the maximum possible steady-state lateral

*Corresponding author, e-mail: matthijs.klomp@saab.com 
acceleration, and the understeer coefficient of the vehicle. These characteristics are of particular interest here since the lateral grip determines the path-keeping capability for the vehicle and the understeer determines the relation between the driver's input (steering) and the achieved cornering radius. The grip limit is an important reference for safe operation of the vehicle and the understeer can give feedback to drivers and indicate how close they are to the grip limit.

One standard method to determine the lateral grip and understeer of a vehicle is the ISO4138:2004 circular driving test 3. Other methods to obtain these results are the handling diagram described in [9], and the MMM-diagram in [6]. Although these methods are intended for constant longitudinal speed, i.e. small drive forces, 9] briefly discusses the implications of constant longitudinal acceleration in the development of the handling diagram. Others, such as 1 and 10, further confirm that the concepts of understeer and lateral grip also hold in the case of moderate levels of constant acceleration or braking, as is also assumed in this study. These methods have been proven to be effective in evaluating the handling characteristics of a vehicle for a fixed longitudinal acceleration. However, since these results need to be recalculated for different drive force distributions, their use for evaluating the effect of drive force distribution is limited. More recent studies, such as that performed by 2, propose an expansion of the handling diagram to a so-called handling surface. However, their paper was limited to the case of a locked differential with the longitudinal slip on the rear axle as a second independent variable. The implications of other differentials such as left/right torque vectoring units and variable front/rear drive force distribution were not discussed.

Based on this review and the importance of lateral grip and understeer, there appears to be a need for improved theoretical descriptions of the influence of the drive force distribution on the lateral grip and understeer. In order to be useful for evaluation of different driveline configurations, the results from these descriptions should be possible to be presented graphically in a meaningful way. One such graphical method is the so-called Dynamic Square, see [4. This method is used to evaluate the influence of the front/rear drive force distribution on the lateral grip, which is of particular interest here.

The objective of this study is to extend the Dynamic Square method so that both the grip limit and understeer can be analyzed under conditions with constant longitudinal acceleration. The goal is to describe the AWD vehicle dynamics with the simplest vehicle model, the single track - or bicycle - model. To facilitate a better correlation of the single-track model to more complex models, closed-form functions that better describe critical axle performance parameters than currently used approximations, is also desired. The fulfillment of these objectives shall be applied to the Dynamic Square graph and produce tools for the analysis and control of a wide range of driveline configurations.

This paper is organized such that first the vehicle model is presented with novel approximations of the axle characteristics of interest. Next the Dynamic Square method is extended and subsequently applied for the analysis and control of four different driveline configurations which together cover the entire range of possible drive force distributions. Lastly the findings are summarized and conclusions are drawn with respect to the obtained results. In the Appendix the vehicle data, used for the performed simulations, is presented. 


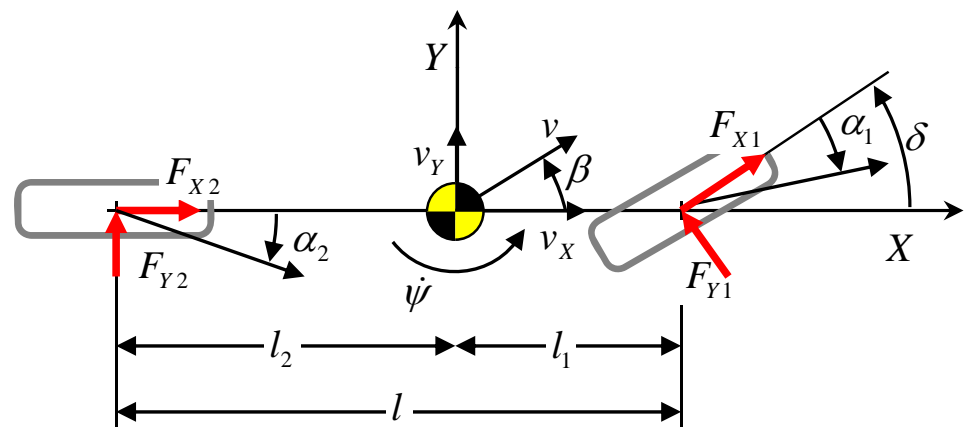

Figure 1: The bicycle vehicle model

\section{Tools for Analyzing the Effect of Drive Force Distribution}

For the purpose of analyzing the front/rear drive force distribution on vehicle performance, a simple bicycle model as shown in Figure 1 is chosen. The choice of a single-track (bicycle) model over a more common two-track model is to facilitate a clearer insight in the interaction between front/rear drive force distribution and the studied properties. The most significant shortcoming of the bicycle model relative to a two-track vehicle model is that the lateral load transfer is not considered. The lateral load transfer is important in this context since the left/right drive forces are (in this study) equal on the left/right wheels, whereas the vertical forces are not equal. Therefore a modified expression for the lateral capacity of each axle is developed to account for changing normal loads. The assumption of equal left/right drive force is relevant for vehicles that have a standard open differential that split the drive force equally to each wheel.

Since the studied handling characteristics, lateral grip and understeer, are only valid for steady-state conditions, the developed vehicle model needs to be valid only for these cases. As is done in [1, it is assumed that the steady-state characteristics can be extended also for the case where the longitudinal acceleration is constant. This assumption leads to the quasi steady state model of the vehicle dynamics, meaning that the longitudinal velocity, $v_{X}$, is assumed to be a slowly varying known parameter. For simplicity, we neglect any aerodynamic or other resistance forces, and assume that the steer angle, $\delta$, is small. The quasi-steady state model can therefore be expressed as

$$
\left[\begin{array}{c}
m a_{X} \\
m a_{Y} \\
0
\end{array}\right]=\left[\begin{array}{c}
F_{X 1}-F_{Y 1} \delta+F_{X 2} \\
F_{Y 1}+F_{X 1} \delta+F_{Y 2} \\
l_{2}\left(F_{Y 1}+F_{X 1} \delta\right)-l_{2} F_{Y 2}
\end{array}\right]
$$

where $m$ is the vehicle mass, and $a_{X}$ and $a_{Y}$ the (quasi steady state) longitudinal and lateral acceleration, respectively. If the path radius is sufficiently large it follows that $\delta \approx 0$. From Equation (1) it is then possible to express the lateral acceleration as function of the lateral force of each respective axle such that

$$
a_{Y}=\frac{l}{m l_{2}} F_{Y 1}=\frac{l}{m l_{1}} F_{Y 2}
$$


The lateral grip, $a_{Y}^{\lim }$, is determined by the front axle (when $F_{Y 1}=F_{Y 1}^{\lim }$ ) and/or the rear axle (when $F_{Y 2}=F_{Y 2}^{\lim }$ ), where $F_{Y}^{\lim }$ is the maximum lateral grip of the front or rear axle for a given longitudinal force. Combining this insight with Equation (2) the important result is achieved that

$$
a_{Y}^{\lim }=\frac{l}{m} \min \left(\frac{F_{Y 1}^{\lim }}{l_{2}}, \frac{F_{Y 2}^{\lim }}{l_{1}}\right) .
$$

Since we would like to express $a_{Y}^{\lim }$ using a bicycle model for a vehicle with open differentials, we need to express the influence of $F_{X}$ on $F_{Y}^{\lim }$ on the level of each axle. Employing the friction circle concept (isotropic tire characteristics), the lateral grip is given by the sum of the left/right wheels:

$$
F_{Y i}^{\lim }=\sum_{j=1}^{2} \sqrt{\mu_{i}^{2} F_{Z i j}^{2}-\left(F_{X i} / 2\right)^{2}}, \quad i=1,2, \quad j=1,2,
$$

where indexes $i=1 / i=2$ and $j=1 / j=2$ are for the front/rear and left/right tires, respectively. The vertical forces on each wheel, $F_{Z i j}$, are given by the static load distribution as well as the longitudinal and lateral load transfer [5, 9]:

$$
F_{Z i j}=m \frac{\left(l-l_{i}\right) g+(-1)^{i} h a_{X}}{2 l}+(-1)^{j} \zeta_{i} m a_{Y}, \quad i=1,2, \quad j=1,2,
$$

where $h$ is the height of the center of mass above the ground and $g$ is the gravitational acceleration and where

$$
F_{X}^{\mathrm{IN}}=F_{X 1}+F_{X 2}
$$

Further, the lateral load transfer coefficient, $\zeta_{i}$, is a lumped parameter combining the effects of the front/rear roll stiffness distribution, roll center heights and height of the mass center.

By combining Equations (2), (4) and (5) we obtain that

$$
F_{Y i}^{\lim }= \begin{cases}\sqrt{\left(\mu_{i} F_{Z i}\right)^{2}-F_{X i}^{2} /\left(1-\theta_{i}^{2}\right)}, & \text { if }\left|F_{X i}\right| \leq \mu_{i} F_{Z i}\left(1-\theta_{i}^{2}\right) \\ \left(\mu_{i} F_{Z i}-\left|F_{X i}\right|\right) / \theta, & \text { otherwise }\end{cases}
$$

where $\theta_{i}=2 \mu_{i} \zeta_{i} l /\left(l-l_{i}\right)$. In Equation (7) we have an expression of the lateral grip for each axle where the individual left/right wheel forces do not need to be considered, which was our aim.

The transition from the quadratic to the linear expression in Equation (7) is physically explained by that for small $F_{X}$ both the inner and outer wheels contribute to the lateral grip. For $\left|F_{X}\right|>\mu F_{Z}\left(1-\theta^{2}\right)$, only the outer wheel contributes to the lateral grip. This transition is illustrated in Figure 2 for a left turn. The two cases where $F_{X}=0$ and where $F_{X}=\mu F_{Z}$ are illustrated in Figures 2 a and 2, c, respectively. In Figure 2 b the transition from quadratic to linear relation between $F_{Y}^{\lim }$ and $F_{X}$ as given by Equation (7) is illustrated. The size of the left/right friction circles as shown in Figure 2 decreases with reduced lateral grip as can be seen in Equation (5).

It may be noted that if $\theta=0$, i.e. no load transfer, Equation (7) reduces to a friction circle such that

$$
\left.F_{Y i}^{\lim _{Y}}\right|_{\theta_{i}=0}=\sqrt{\mu_{i}^{2} F_{Z i}^{2}-F_{X i}^{2}}, \quad i=1,2 .
$$



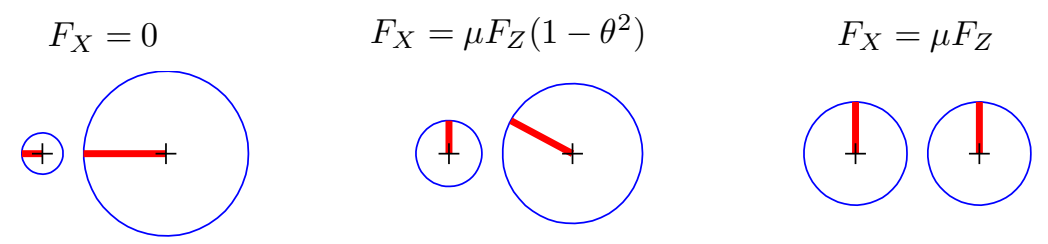

Figure 2: Fully utilizing the available lateral grip for three different levels of $F_{X}$. The force vector is indicated by the bold line and the friction capacity for the left and right wheels are shown with circles.

The result in Equation (7) still requires the knowledge of the lateral load transfer coefficient $\theta$. Additionally, two expressions are required to describe one vehicle parameter depending the drive force. The most common approximation proposed in the literature (see [8]) is to use Equation (8) also for $\theta>0$. However, here a different approximation is proposed:

$$
\tilde{F}_{Y i}^{\lim }=\mu_{i}^{2} F_{Z i}^{2}-F_{X i}^{2}, \quad i=1,2,
$$

which also allows for direct estimation of $F_{Y i}^{\lim }$ as with Equation (8).

Naturally, if $\theta_{i}$ is small, Equation (8) is a good approximation of Equation (7). The question is for which load transfer coefficient, $\theta_{i}^{*}$, is Equation (7) best approximated with the proposed method in Equation (9)? This $\theta_{i}^{*}$ is found by solving the least-squares problem

$$
\min _{\theta_{i}}\left\|\int_{0}^{\mu_{i} F_{Z i}}\left(F_{Y i}^{\lim }-\tilde{F}_{Y i}^{\lim }\right) \partial F_{X i}\right\|_{2}^{2}, \quad i=1,2 .
$$

The numerical value of $\theta_{i}$ that minimizes Equation 10 is $\theta_{i}^{*} \approx 0.6121$. This value is somewhere between the lateral load transfer of front and rear axle of the vehicle which is studied here (Appendix A) which can also be seen in Figure 3 . There it can be seen that Equation (9) is a good approximation of the lateral grip on the front axle especially, although it overestimates the grip on the rear axle. For this particular vehicle configuration, Equation (9) can in Figure 3 be seen to be a better approximation than the friction circle Equation (8).

If the friction coefficient, $\mu$, and the drive force distribution are known then Equation (3) and Equation (9) provides the basic tool to compute the lateral grip and, as will be seen below, also the vehicle understeer.

\section{Lateral Grip and Understeer Presented in the Dynamic Square}

In order to evaluate various combinations of the front/rear drive force distributions on the cornering performance, the so-called Dynamic Square described by [4] is used. As shown in Figure 4 the lateral grip $a_{Y}^{\lim }$, determined in Equation (3), is given as a level plot as function of the longitudinal force on the front and rear axle. All numerical examples in this section use vehicle data from Appendix A. 


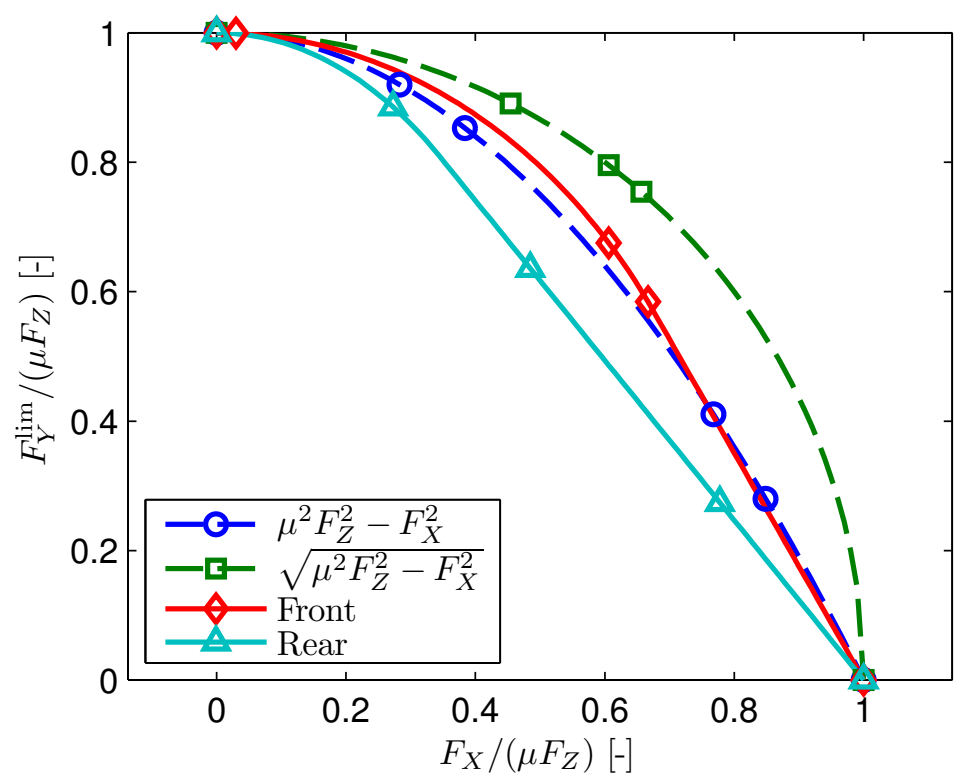

Figure 3: Normalized lateral grip per axle versus normalized longitudinal force divided equally on the left and right wheels. The lateral grip using data from Appendix $\mathrm{A}$ is compared with two different approximations shown with dashed curves.

As can be seen from Equation (3), the front axle limits the lateral grip when $l_{1} F_{Y 1}^{\lim }<l_{2} F_{Y 2}^{\lim }$, i.e. when the maximum yaw moment from the front axle is less than from the rear axle. This is represented by the dark grey area in Figure 4 .a. Conversely the light grey area is where the rear axle saturates first, i.e. where $l_{1} F_{Y 1}^{\lim }>l_{2} F_{Y 2}^{\lim }$, and which therefore represents an area where the vehicle can become unstable. Along the line separating the two areas $l_{1} F_{Y 1}^{\lim }=l_{2} F_{Y 2}$. This is the line where both axles saturate simultaneously and thus represents the maximum lateral grip for a given $F_{X}^{\mathrm{IN}}$.

The plot in Figure 4 a differs from the plot in [4] as the separation line between the front and rear axle saturation areas does not pass through the origin. The reason for this is that the total friction capability is not assumed to be equal on the front and rear axles as is the case in 4 4. Also, the results presented in Figure 4 a uses the expression Equation 97 for computing the lateral grip since it better approximates the influence of the longitudinal force on the friction capability than a friction circle, as discussed in Section 2. The applications of these new equations in the Dynamic Square produce results consistent with the MMM-diagram [6] but in a more convenient form, since the study only needs a single graph.

The contours describing constant levels of $a_{Y}^{\lim }$ are provided in Figure 4. a. The global maximum occurs for a negative value of $F_{X 1}$. This is a consequence of the static forward weight bias of the vehicle in the expression for $a_{Y}^{\lim }$.

For the study of the effect of the front/rear drive force distribution on the understeer, the well-known expression for the understeer [9] relating the cornering 
(a)

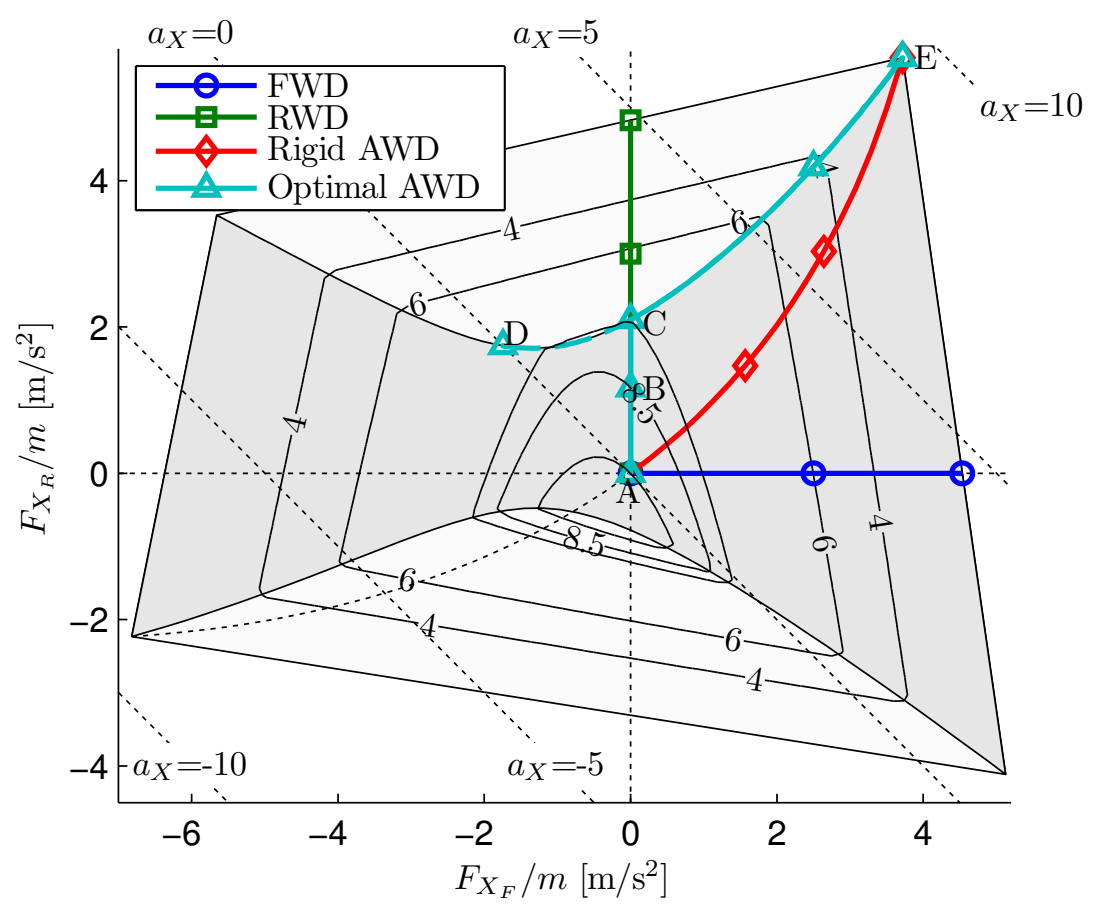

(b)

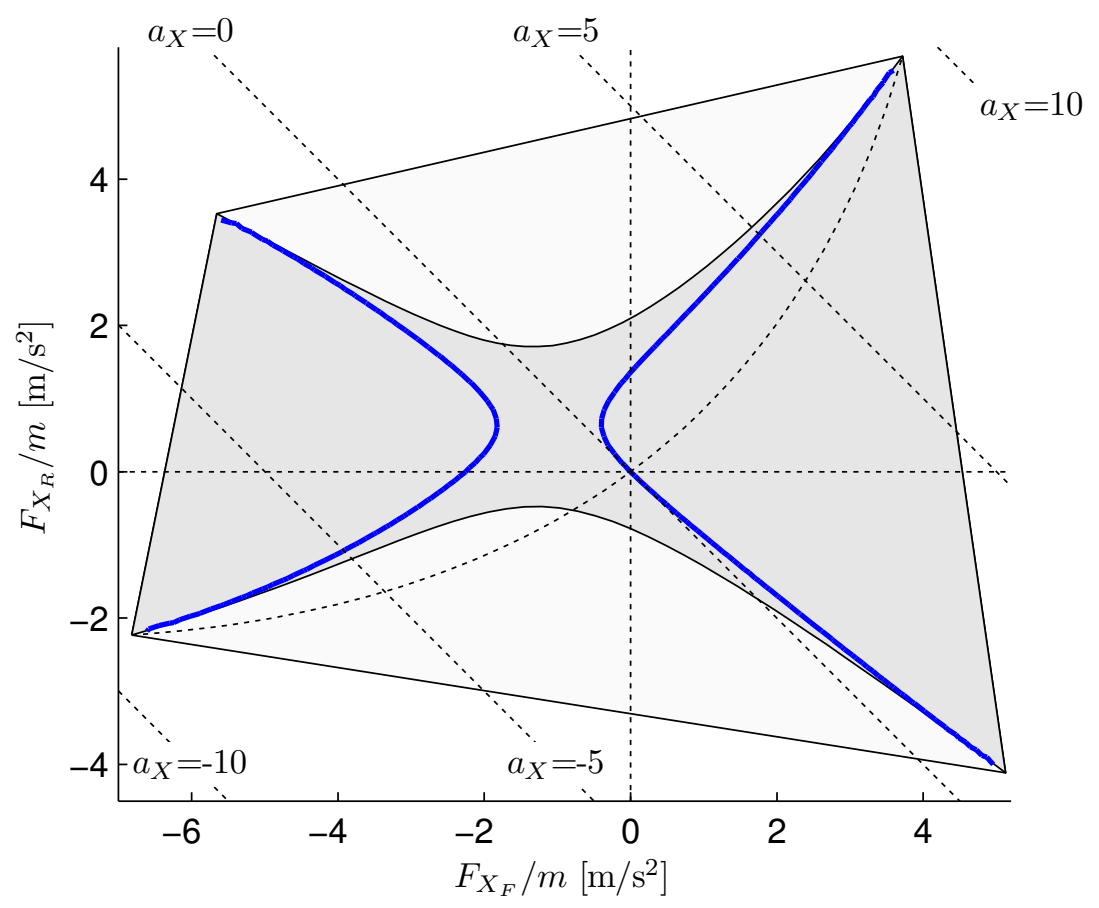

Figure 4: Influence of the front/rear drive force distribution on the lateral grip, $a_{Y}^{\lim }$, in (a) and the understeer, $K_{u}$, in (b). The front axle first saturates in the dark grey areas and the rear axle saturates first in the light grey areas. The level curves in (a) show constant levels of lateral grip. Along the bold curves in (b) the understeer is constant. 
stiffness and vehicle parameters is

$$
K_{u}=-\frac{m}{l} \frac{l_{1} C_{\alpha 1}-l_{2} C_{\alpha 2}}{C_{\alpha 1} C_{\alpha 2}} .
$$

If $C_{\alpha}$ is the cornering stiffness for zero drive force (see Appendix A) then $C_{\alpha}^{\prime}$ can be replaced by the effective cornering stiffness for a given drive force. The change in cornering stiffness as function of drive force is modeled using Equation (9) such that

$$
C_{\alpha}^{\prime}=C_{\alpha} \frac{F_{Y}^{\lim }}{\mu F_{Z}}=C_{\alpha}\left(1-\left(\frac{F_{X}}{\mu F_{Z}}\right)^{2}\right) .
$$

By using the vertical forces $(5)$ and by using that $C_{\alpha}$ is the cornering stiffness at the static vertical force it follows that

$$
C_{\alpha 1}^{\prime}=C_{\alpha 1}\left(1-\frac{h}{l} \frac{a_{X}}{m}\right)\left(1-\left(\frac{F_{X 1}}{\mu_{1} F_{Z 1}}\right)^{2}\right) \approx C_{\alpha 1}\left(1-\frac{h}{l} \frac{a_{X}}{m}\right),
$$

if $F_{X 1} \ll \mu_{1} F_{Z 1}$ and similarly

$$
C_{\alpha 2}^{\prime} \approx C_{\alpha 1}\left(1+\frac{h}{l} \frac{a_{X}}{m}\right) .
$$

By replacing the cornering stiffness, $C_{\alpha}$, in Equation (11) with the approximations of the effective cornering stiffness, $C_{\alpha}^{\prime}$ given by Equations (13) and (14), we obtain that

$$
K_{u}=-m g \frac{l_{1} C_{\alpha 1}\left(l g-h a_{X}\right)-l_{2} C_{\alpha 2}\left(l g+h a_{X}\right)}{C_{\alpha 1} C_{\alpha 2}\left(l^{2} g^{2}-h^{2} a_{X}^{2}\right)} .
$$

This modified expression for the understeer is similar to the results presented in [9, but expressed using the same vehicle parameters used in the previous modeling section. The expression can be used to explain why a vehicle can become oversteered when braking $\left(a_{X}<0\right)$ and that acceleration $\left(a_{X}>0\right)$ explicitly increases understeer.

Even though the above result is a very simple way to take into account the longitudinal load transfer caused by acceleration and braking, it does not take into account the drive force distribution. Since the Dynamic Square method is an effective way to show the influence of the drive force distribution on the lateral grip; the idea is that it can also be used to show the effect on the understeer. Thus the understeer gradient Equation (11) is shown as level plots for all possible combinations of front/rear drive force distributions in Figure 4 . $\mathrm{b}$ The understeer gradient is shown under the assumption that the cornering stiffness changes according to Equation (12).

In Figure $4 \mathrm{~b}$ the results of this modified Dynamic Square can be seen. The diagram shows the effect of the rear wheels saturating along the top- and bottom edges. Along these edges, $K_{u} \rightarrow-\infty$ as $C_{\alpha 2}^{\prime} \rightarrow 0$ because $F_{X 2} \rightarrow \mu_{2} F_{Z 2}$; similarly, along the left and right edges where the front wheels saturate, $K_{u} \rightarrow$ $\infty$ as $C_{\alpha 1}^{\prime} \rightarrow 0$ when $F_{X 1} \rightarrow \mu_{1} F_{Z 1}$. Further, the diagram is divided into three different areas; the light-grey areas at the top and bottom are areas where the understeer gradient is negative, i.e. oversteeer; conversely, the dark grey areas indicate (positive) understeer. Based on these descriptions, the curves between the dark and light areas mark the neutral steer boundaries, i.e. where $K_{u}=0$. 
The two versions of the Dynamic Square in Figures 4 a and 4 . b share related information. The light grey areas reflect conditions where the rear axle saturates first promoting oversteer. Conversely the dark areas indicate the understeer characteristics which is the result of a saturated front axle. Further discussion of drive force distribution on cornering performance will be limited to the grip margin as similar conclusions will apply to the understeer properties.

\section{Particular Driveline Configurations and Vehi- cle Handling}

In order to illustrate how the results derived in the previous sections can be applied to the analysis of the influence of lateral grip and understeer of an arbitrary drive force distribution, these effects are in this section shown by means of the so-called Dynamic Square diagram from 4]. These diagrams are level plots of the lateral grip or understeer versus the drive force distribution and an added feature of the Dynamic Square is that the optimal drive force distribution can be graphically identified. Optimality is defined as the drive force distribution that results in the maximum lateral grip possible for a given total drive force. Further G-G diagrams, which are described in [6], are shown in Figure 5 and which are traditionally used to investigate the effects of front, rear, and rigid all wheel drive systems on combined lateral and longitudinal grip. A rigid all-wheel drive system is defined as a system where the front and rear differentials rotate synchronously. In order to aid comparison, the Dynamic Square in Figure 4 show curves with legends that correspond to the G-G diagrams in Figure 5 .

To further investigate the application of the Dynamic Square for vehicle control, expressions relating the total drive force to the lateral grip for three reference drivelines are developed. In all cases the analysis is limited to cases with positive longitudinal (drive) forces available in AWD systems. Generalization of the method is possible for negative longitudinal (braking) forces but are not addressed here. Only the first quadrant of the Dynamic Square diagram (Figure 4) is considered in the following cases.

For future reference, the front/rear drive force distribution is defined as:

$$
\xi=\frac{F_{X 1}-F_{X 2}}{F_{X}^{\mathrm{IN}}}
$$

\subsection{Front-Wheel Drive}

For a FWD vehicle it follows that $\left.F_{X}^{\mathrm{IN}}\right|_{\xi=1}=F_{X 1}$. From Equation (3) it can be seen that the front axle will always saturate prior to the rear if $\mu_{1}>\mu_{2}$, which gives that

$$
a_{Y}^{\lim , \mathrm{FWD}}=\left.\frac{l}{m l_{2}} F_{Y 1}^{\lim }\right|_{\xi=1}, \quad \forall F_{X 1} \in\left[0, \frac{\mu_{1} m g l_{2}}{l+h \mu_{1}}\right] .
$$

The condition on the magnitude of $F_{X 1}$ is due to $F_{X 1} \leq \mu_{1} F_{Z 1}$. 


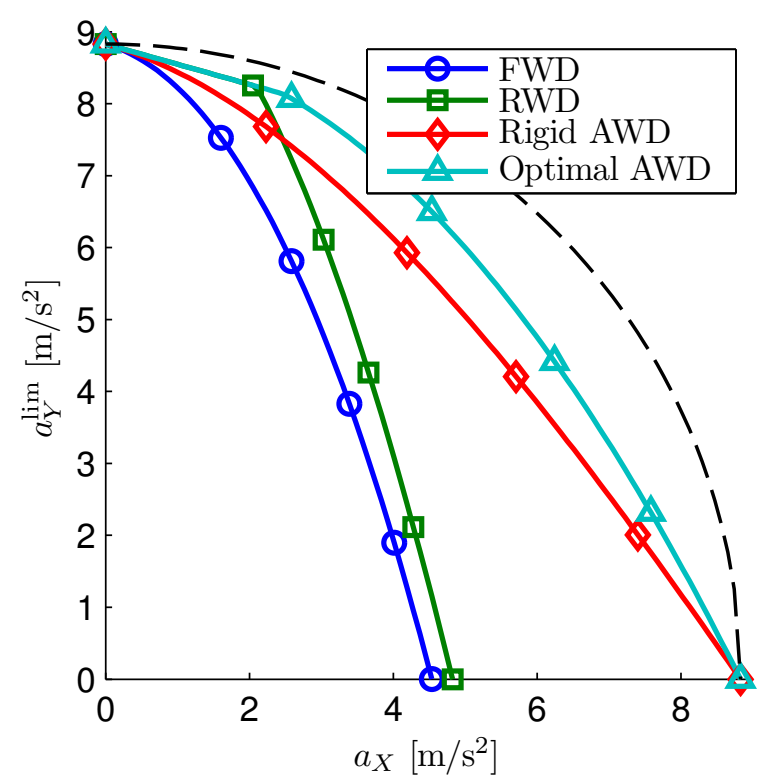

Figure 5: G-G diagrams for four different drive force distributions. These are sections through the Dynamic Square indicated with corresponding curves in Figure 4 a

\subsection{Rear-Wheel Drive}

Analogous to the FWD case, it is possible to derive $a_{Y}^{\lim }$ for a RWD vehicle from Equation (3)

$$
a_{Y}^{\lim , \mathrm{RWD}}=\frac{l}{m} \min \left(\frac{\mu_{1} F_{Z 1}}{l_{2}}, \frac{\left.F_{Y 2}^{\lim }\right|_{\xi=-1}}{l_{1}}\right) . \quad \forall F_{X 2} \in\left[0, \frac{\mu_{2} m g l_{1}}{l-h \mu_{2}}\right] .
$$

The main difference between Equations $(17)$ and $(18)$ is that the front axle limits the lateral grip for small $F_{X 2}$. This is always the case for the FWD vehicle, but a RWD vehicle transitions from the front axle limiting the grip for small $F_{X 2}$ to the rear axle limiting the lateral grip for large $F_{X 2}$.

\subsection{Rigid All-Wheel Drive}

For a rigid all-wheel drive configuration the front and rear axles rotate at the same speed. Since the traction stiffness of the tires is approximately proportional to the normal load on the tires (see [9]), the drive force distribution then follows the vertical force distribution in Equation (5), i.e.

$$
\xi^{\mathrm{rAWD}}=\frac{F_{Z 1}-F_{Z 2}}{F_{Z 1}+F_{Z 2}}=-\frac{l_{1}-l_{2}}{l}-\frac{2 h F_{X}^{\mathrm{IN}}}{m g l} .
$$

For stable operation it is desirable that the vehicle performance is restricted to the case where the front axle saturates first. This applies to the area in Figure 4 where $F_{X}^{\mathrm{IN}}<\mu_{1} F_{Z 1}+\mu_{2} F_{Z 2}$ where the front axle limits the lateral grip for this 
configuration. Using this constraint, the lateral grip, Equation (3) reduces to

$$
a_{Y}^{\lim , \mathrm{rAWD}}=\left.\frac{l}{m l_{2}} F_{Y 1}^{\lim }\right|_{\xi=\xi^{\mathrm{rAWD}}}, \quad \forall F_{X}^{\mathrm{IN}} \in\left[0, m g \frac{\mu_{1} l_{1}+\mu_{2} l_{2}}{l}\right] .
$$

The lateral grip in Equation 20 with the drive force distribution given by Equation (19) shown in Figure 4 as the curve with diamond markers.

\subsection{Optimal Front/Rear Drive Force Distribution}

Since the Dynamic Square shows the lateral grip for the entire solution space of possible combinations of $F_{X 1}$ and $F_{X 2}$ the drive force distribution that gives the maximum lateral grip can be graphically identified. For any given $F_{X}^{\mathrm{IN}}$, which is a diagonal line in the Dynamic Square, the point where this diagonal is tangent to a level curve of the Dynamic Square is the optimal drive force distribution, $\xi^{*}$.

In Figure 4 it can be seen that the maximum lateral grip for large $F_{X}^{\mathrm{IN}}$ is along the border between the dark and light grey areas (where $l_{1} F_{Y 1}^{\lim }=l_{2} F_{Y 2}$ ). This is the optimal drive force distribution with the exception for $F_{X}^{\mathrm{IN}}: F_{X 1} F_{X 2}<0$, i.e. where not both front and rear drive forces are positive. As can be seen from point B in Figure 4 the constant longitudinal acceleration line (inclined dashed line) is tangent to the level curve where $F_{X 1}=0$. This means that as long as the front axle is limiting the lateral grip, the optimal front/rear drive force distribution is to only drive the rear wheels $(\xi=0)$ up to point $\mathrm{C}$ in the figure. The bold solid line in Figure 4 shows the solution to Equation 21) with the constraint that $F_{X 1}$ must be in the interval of $F_{X 1} \in\left[0, \mu_{1} F_{Z 1}\right]$. The bold dashed line C-D shows the drive force distribution that arises without this constraint, which gives a lower $a_{Y}^{\lim }$ than would be the case if that constraint is added.

The conclusion from the above discussion is that the optimal drive force distribution is

$$
\xi^{*}= \begin{cases}-1, & \text { if } F_{X 1} F_{X 2} \leq 0, \\ \xi: l_{1} F_{Y 1}^{\lim }-l_{2} F_{Y 2}^{\lim }=0, & \text { otherwise }\end{cases}
$$

and is the optimal drive force distribution for that range of total drive force.

\subsection{Control Authority of some Specific Driveline Config- urations}

The true utility of the Dynamic Square approach is observed when investigating the control authority of different physical driveline configurations. The control authority of some driveline configurations which operate between the above special cases by controlling the slippage in one (or two) clutch(es) is described in Figure 6, based on the previously described Dynamic Squares method. Table 1 describes configurations with one clutch. These configurations are of interest since they exist in a number of vehicle applications on the market today. A double clutch configuration is not known to be a configuration existing on any production vehicle, but is interesting since it operates between three different "modes" as given by Table 2 and offers maximum drive force distribution flexibility. 

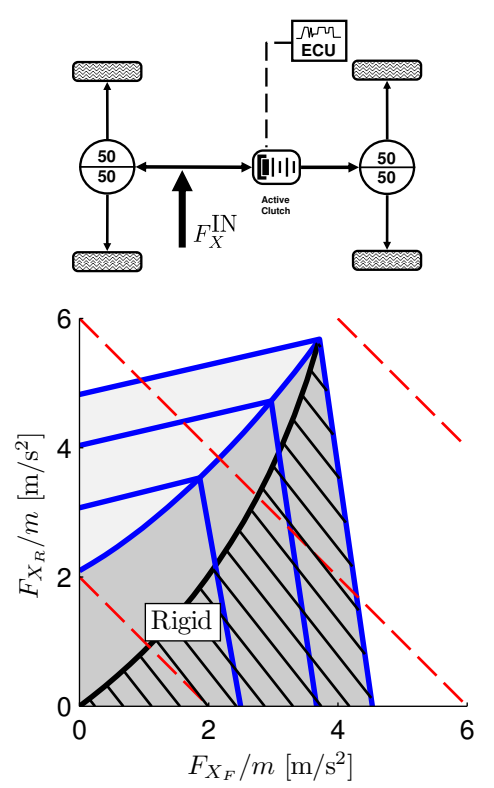

(a) FWD and rear torque transfer control
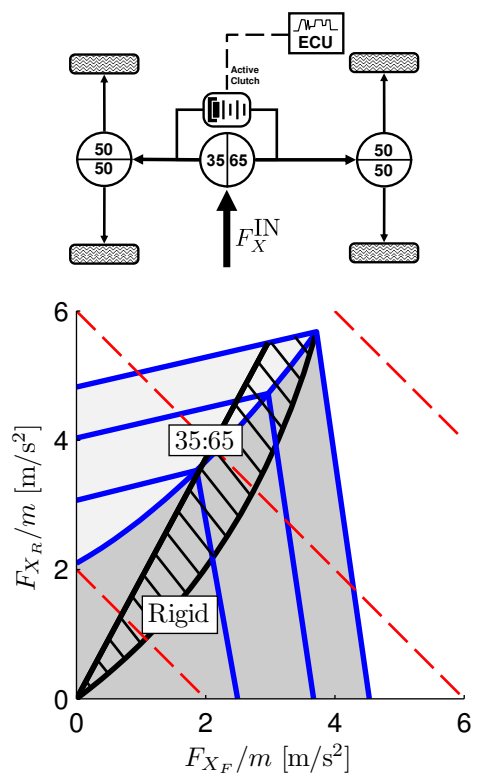

(c) Permanent AWD with active center differential
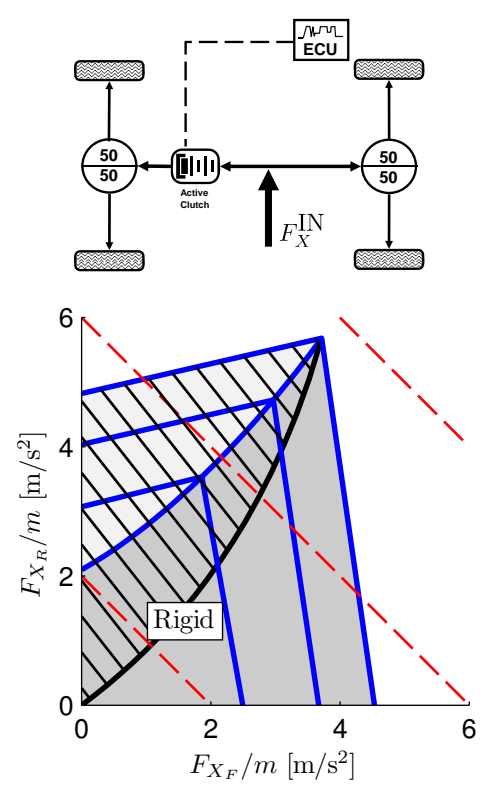

(b) FWD and front torque transfer control
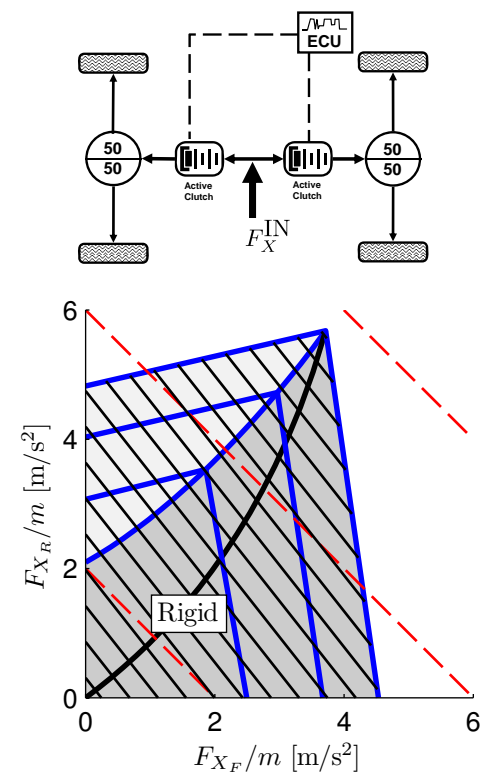

(d) Front and rear axle torque control

Figure 6: Authority shown as a hatched area for four different configurations.

Rigid in the Dynamic Square diagrams means a locked center clutch. 


\begin{tabular}{|l|l|l|}
\hline Description & Clutch open & Clutch locked \\
\hline \hline FWD $\Rightarrow$ Fixed AWD & $\xi=1$ & $\xi=\xi^{\text {rAWD }}$ \\
RWD $\Rightarrow$ Fixed AWD & $\xi=-1$ & $\xi=\xi^{\text {rAWD }}$ \\
$35: 65^{1} \Rightarrow$ Fixed AWD & $\xi=-0.3$ & $\xi^{\text {rAWD }}$ \\
\hline
\end{tabular}

Table 1: Driveline configuration that operate between two different dirve force distributions by controlling a clutch that controls the front/rear drive force distribution.

\begin{tabular}{|l|l|l|}
\hline Front Clutch & Rear Clutch & Drive force distr. \\
\hline Locked & Open & $\xi=1$ \\
Locked & Locked & $\xi=\xi^{\text {rAWD }}$ \\
Open & Locked & $\xi=-1$ \\
\hline
\end{tabular}

Table 2: Driveline configuration that operate between three different operating regions by controlling two clutches

For all configurations in Figure 6 it is important to consider the rigid AWD case which was described in Section 4.3. In all these configurations, locking the front and rear axles together yield the drive force distribution line denoted as "Rigid" in the figures.

From Figure 6. a it can now be seen that a vehicle, which is FWD in it's basic configuration (open clutch), can only operate at an optimal front/rear drive force distribution at the upper right vertex of the diagram. However, this vehicle configuration is not able to accomplish a drive force distribution above the rigid line. The advantage of this system is that it always operates in the safe area where the front axle is limiting the lateral grip.

The configuration shown next in Figure 6. b, is a RWD based vehicle which is the inverse of the previous case and has the authority to optimize the torque transfer to the front axle such that the optimal drive force distribution given by Equation 211) can be achieved. On the other hand, operating in the light grey area where the rear axle is limiting the lateral grip can lead to instability.

The third configuration shown in Figure 6 $\mathrm{c}$ is what often is called permanent AWD, with a center differential providing a fixed front/rear drive force distribution. This configuration is a useful reference since it the most commonly used AWD configuration, when also vehicles without a controllable clutch are considered.

The final configuration, shown in Figure 6 d. is able to operate in the entire first quadrant of the Dynamic Square diagram (however, not in the second, unless the brakes are used) and is therefore an interesting concept. This system combines the benefits of both the configurations in Figure 6.a) and Figure6. b).

\section{Conclusions}

In this paper the influence of longitudinal acceleration and the drive force distribution on the lateral grip and understeer are shown. The effects studied are limited to steady-state analysis with constant longitudinal acceleration, defined as quasi steady-state conditions. 
In order to illustrate the studied effects with as simple mathematical descriptions as possible, a modified way to express the influence of the longitudinal force on the lateral grip of an axle is presented. This method enabled the use of a simple bicycle model as opposed to a more often used two-track model. Expressions for the lateral grip limits and vehicle understeeer coefficients are developed to account for general cases of front/rear drive force distributions. Lateral load transfer is also implemented into the simpler model to more accurately describe the influence of drive forces on a vehicle's dynamic behavior.

Building on these expressions, a graphical method presented in [4] is used to show the effect of an arbitrary front/rear drive force distribution on the lateral grip. Using this diagram the optimal front/rear drive force distribution can be graphically identified. The use of the Dynamic Square method is here further extended to also show the effect on the vehicle understeer. As a result, important information of cornering performance can be presented in a more convenient form than conventional methods (G-G diagram, MMM-diagram, etc) as different drive cases do not require re-calculation and re-plotting. Also, the newly presented way of showing the lateral grip and understeer coefficients in the same format allows for parallel analysis of cornering performance and handling.

Improved performance of AWD vehicles can be achieved if the AWD systems can be exploited to achieve maximum performance and safety if suitable analysis tools are available. The Dynamic Square plots are shown to be effective tools for establishing control strategies for optimal lateral grip performance as well as identifying the available control authority.

\section{Acknowledgments}

The authors would like to acknowledge the financial support from the Swedish national research program "Intelligent Vehicle Safety Systems" (IVSS). We would further like to thank Anders Boström, Bo Egardt, Mathias Lidberg from Chalmers University of Technology and Gunnar Olsson from Saab Automobile for reviewing and providing valuable comments to this work.

\section{References}

[1] Masato Abe. A theoretical analysis on vehicle cornering behaviors in acceleration and in braking. In 9th IAVSD Symposium, Dynamics of Vehicles on Roads and on Tracks, 1986.

[2] F. Frendo, G. Greco, M. Guiggiani, and A. Sponziello. The handling surface: a new perspective in vehicle dynamics. Vehicle System Dynamics, 45(11):10011016, 2007.

[3] ISO Technical Committee TC 22/SC9. Steady-state circular driving behaviour. In $I S O$ 4138, 2004.

[4] Manabu Kato, Keiji Isoda, and Hiroo Yuasa. Study on vehicle dynamics in marginal condition using dynamic square method. In SAE Technical Paper 958503, 1995. SAE-958503.

[5] Matthijs Klomp. On Drive Force Distribution and Road Vehicle Handling - A Study of Understeer and Lateral Grip. Licentiate Thesis, Chalmers University of Technology, 2007. 
[6] William F. Milliken and Douglas L. Milliken. Race Car Vehicle Dynamics. SAE International, 1995. 1-56091-526-9.

[7] Russell P. Osborn and Taehyun Shim. Independent control of all-wheel-drive torque distribution. Vehicle Systems Dynamics, 44(7):529-546, July 2006.

[8] Hans B. Pacejka. Simplified analysis of steady-state behaviour of motor vehicles. Vehicle System Dynamics, 2:161-172, 173-183, 185-204, 1973.

[9] Hans B Pacejka. Tyre and Vehicle Dynamics. Butterworth-Heinemann, Oxford, UK, 2 edition, 2006.

[10] Y. Shibahata, K. Shimada, and T. Tomari. The improvement of vehicle maneuverability by direct yaw moment control. In International Symposium on Advanced Vehicle Control, 1992.

\section{A Vehicle Data}

The vehicle data shown in Table 3 represent a medium-sized passenger vehicle.

\begin{tabular}{|l|c|c|c|}
\hline Description & Variable(s) & Unit & Value(s) \\
\hline \hline Vehicle mass & $m$ & {$[\mathrm{~kg}]$} & 1500 \\
\hline Wheel base & $l$ & {$[\mathrm{~m}]$} & 2.675 \\
\hline Height of mass center & $h$ & {$[\mathrm{~m}]$} & 0.5 \\
\hline Axle to mass center distance (front/rear) & $l_{1} / l_{2}$ & {$[\mathrm{~m}]$} & $0.4 l / 0.6 l$ \\
\hline Lateral load transfer coefficients (f/r) & $\zeta_{1} / \zeta_{2}$ & {$[-]$} & $0.17 / 0.16$ \\
\hline Friction coefficients (f/r) & $\mu_{1} / \mu_{2}$ & {$[-]$} & $0.90 / 1.0$ \\
\hline
\end{tabular}

Table 3: Vehicle Data 